\title{
Mapping the 8-Aminolaevulinic Acid Synthetase Locus in Bacillus subtilis
}

\author{
By I. KISS, I. BEREK AND G. IVÁNOVICS \\ Institute of Microbiology, Medical University, Szeged, Hungary \\ (Accepted for publication 20 March 1971).
}

\begin{abstract}
SUMMARY
HemA mutants of Bacillus subtilis I68 requiring $\delta$-aminolaevulinic acid for their growth were isolated either following selection with streptomycin or by exposure to copper sulphate, and studied in reciprocal transduction experiments. Both groups of mutants were found to be genetically identical and the hemA locus was unlinked to a streptomycin resistance marker; it was linked to the group III markers mapped by Dubnau, Goldthwaite, Smith \& Marmur (1967) and is located between the ilvAI and phe-r loci.
\end{abstract}

\section{INTRODUCTION}

Bacillus subtilis strain 168 exposed to either copper sulphate or streptomycin gives mutants defective in protohaem synthesis (Anderson \& Ivánovics, 1967). For routine isolation of such mutants streptomycin selection was convenient and effective (Marjai, Kiss \& Ivánovics, 1970). One class of mutants (hem $A$ ) required $\delta$-aminolaevulinic acid (ALA) for growth and were apparently deficient in $\delta$-aminolaevulinic acid synthetase activity. The aim of this work was to determine the location of the hemA locus in the genome of Bacillus subtilis I 68 in relation to the genetical markers described by Dubnau et al. (1967).

\section{METHODS}

Bacterial strains. The results of preliminary experiments suggested that hem $A$ was located somewhere in the third linkage group of Dubnau et al. (I967). Only strains with reference markers in this linkage group and their hem $A$ derivatives obtained by transformation were therefore used. Table I lists the strains used for mapping the hemA locus, except for a number of mutants with ALA requirement which were isolated from SZ I and from a streptomycin-resistant derivative of it and used in experiments for the characterization of the hemA locus. These strains, try-2hemA2str, try-2hemAI6str, and try-2hemAI7str were isolated from a mutant resistant to $>500 \mu \mathrm{g}$. $/ \mathrm{ml}$. streptomycin following exposure to copper sulphate. Strains try-2hemA6 and try-2hemA8 were obtained by streptomycin selection (Marjai et al. 1970).

Phages. Phage 3 NT (Ivánovics \& Csiszár, 1962) and PBS-I (Takahashi, I96I) were used for transduction. Lysates were prepared and assayed as described previously (Csiszár \& Ivánovics, I965).

Media. Yeast extract peptone medium (YP) was the complete nutrient medium. Glutamate glycerol medium (GGM) was prepared as described by Anderson \& Ivánovics (1967), and supplemented with specific growth requirements when appropriate: $2 \mu \mathrm{g} . / \mathrm{ml}$. ALA, $50 \mu \mathrm{g}$. $/ \mathrm{ml}$. tryptophan and $30 \mu \mathrm{g}$. $/ \mathrm{ml}$. other amino acids. Solid media contained $\mathrm{I} \cdot 5 \%(\mathrm{w} / \mathrm{v})$ agar. 
Transduction. Recipient bacteria grown overnight in YP medium were diluted $\mathrm{I}: 50$ in the same medium, aerated by shaking in a water bath at $37^{\circ}$, and grown to an extinction of 0.45 at $620 \mathrm{~nm}$. equivalent to $2 \times 10^{8}$ bacteria $/ \mathrm{ml}$. For hem $A$ mutants YP medium was supplemented with ALA. Transducing phage was added at a multiplicity of infection of one, the mixture kept at room temperature for $30 \mathrm{~min}$. and then centrifuged for $15 \mathrm{~min}$. at $2500 \mathrm{rev} . \mathrm{min}$. in an angle centrifuge, the pellet was then resuspended in the same volume of GGM. Recombinants were selected on appropriately supplemented GGM plates and scored for unselected markers by replica plating to other media either directly or following restreaking on selective medium (both methods gave identical results).

Table I. Strains used to map the hemA locus in Bacillus subtilis 168

\begin{tabular}{|c|c|c|}
\hline Strain & Previous name & Genotype \\
\hline SZI & $\mathrm{RI} 2$ & $\operatorname{try}-2$ \\
\hline$\$ Z 2$ & - & Prototroph \\
\hline$s \angle 3$ & RI 5 & $\operatorname{try}-2$ hem $A I$ \\
\hline SZ. 4 & GSY 384 & $\arg A 2 \operatorname{leu}-I$ \\
\hline$S \angle 5^{*}$ & - & $\arg A 2$ leu-I hem $A I$ \\
\hline $\operatorname{sz} 6 *$ & — & $\arg A 2$ hem $A I$ \\
\hline$s Z 7^{*}$ & - & leu-I hemAI \\
\hline sz8 & GSY 244 & phe-I ilvAI \\
\hline $\operatorname{szg} 9^{*}$ & - & phe-I hemAI \\
\hline$S \angle 10^{*}$ & - & ilvAI hemAI \\
\hline SZII & - & try-2 str \\
\hline $\mathrm{SZ} \mathbf{I} 2$ & -- & try-2hemAI str \\
\hline SZ 13 & -- & try-2 nia-6o \\
\hline sZ. $14^{*}$ & -.. & hemAI \\
\hline
\end{tabular}

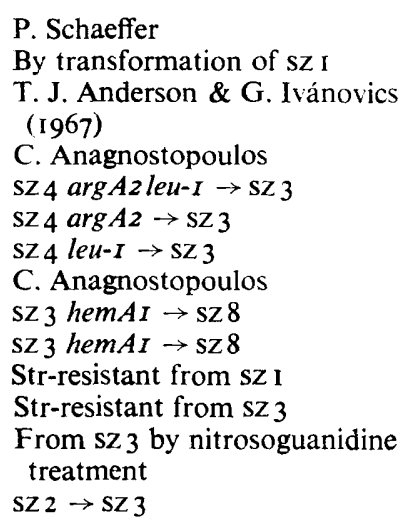

* Strains constructed by transformation of the strains indicated. The arrows indicate the relevant donors.

\section{Analysis of the hemA locus}

RESULTS

The copper sulphate method only yielded mutants with a protohaem deficiency when a streptomycin-resistant mutant of strain 168 was used. So far, all attempts to obtain such mutants from streptomycin-sensitive bacteria have failed. When haem mutants were isolated from streptomycin-sensitive I68 bacteria following streptomycin selection, a fairly high concentration (100 to $200 \mu \mathrm{g} . / \mathrm{ml}$.) was used. Nevertheless, the haem dependency was associated with only a small increase in the streptomycin resistance of the mutants which was lost upon spontaneous reversion to haem independency (Marjai et al. 1960). The mutants could not be classed as conditionally streptomycin-dependent (Gorini \& Kataja, 1964), since streptomycin did not substitute for the requirement of the auxotroph. It therefore seemed likely that the haem requirement was not associated with an alteration of the streptomycin locus, although the observations referred to above might indicate some relation between streptomycin and haem markers.

To test whether the str and hemAI markers were linked or not, strain Sz3 (try-2hemAI) was transduced with phage 3 NT propagated on strain SZ I I $\left(\right.$ try-2str) and the selected hem $A I^{+}$ transductants screened for streptomycin resistance by replication. None of 800 colonies was streptomycin-resistant. Also, when strain SZ I was transduced with SZI2 lysates no cotransduction of str and hemAI markers was obtained. In this case, the transductants were selected for streptomycin resistance on plates containing tryptophan, ALA, + streptomycin, and replicated to GGM plates containing only tryptophan. 
In reciprocal transduction crosses made with hemA mutants isolated either by copper sulphate or streptomycin treatment very few or no transductants were obtained. These results indicate that a single gene codes for $\delta$-aminolaevulinic acid synthetase.

\section{Mapping of the hemA locus}

Two-factor crosses. Since one of our aims was to use phage 3 NT instead of PBS-I and our transduction technique was not the same as that of Dubnau et al. (1967), transduction with the two phages was compared using the technique developed in this laboratory (Csiszár \& Ivánovics, 1965). All recipients used possessed the standard markers of Dubnau et al. (1967). Both phages, PBS-I and 3 NT, gave identical results (see Table 2). Hoch, Barat \& Anagnostopoulos (1967) also obtained the same results in comparative transduction tests with these two phages and recombination-defective mutants of Bacillus subtilis. Recombination frequencies were identical for all markers tested, and corresponded well with the values obtained by Dubnau et al. (1967) except for $l e u^{+}$transductants of sz 4. On minimal medium supplemented only with arginine, the frequency of recombinants did not exceed 10 \%, but on the sequential addition of amino acids except leucine to GGM+arginine there was a gradual increase in recombination which reached a peak value of $65.4 \%$ when all (19) amino acids had been added.

Table 2. Recombination between standard reference markers in transduction with phages $3 N T$ and $P B S-\mathrm{I}$

\begin{tabular}{|c|c|c|c|c|c|c|}
\hline \multirow[b]{2}{*}{ Recipient } & \multirow[b]{2}{*}{ Phage* } & \multirow[b]{2}{*}{$\begin{array}{l}\text { Selected } \\
\text { marker }\end{array}$} & \multirow[b]{2}{*}{$\begin{array}{l}\text { Total no. of } \\
\text { transductants }\end{array}$} & \multicolumn{3}{|c|}{ Recombinants } \\
\hline & & & & Class & $\begin{array}{l}\text { No. of } \\
\text { colonies }\end{array}$ & $\%$ \\
\hline \multirow[t]{2}{*}{ sz 8 (phe-I ilvAI) } & $3 \mathrm{NT}$ & $\begin{array}{l}i l v^{+} \\
\text {phe }\end{array}$ & $\begin{array}{l}\text { I } 547 \\
\text { I } 579\end{array}$ & $\begin{array}{l}\text { pheilv+ } \\
\text { phe } e^{+} i l v\end{array}$ & $\begin{array}{l}342 \\
38 \mathrm{I}\end{array}$ & $\begin{array}{l}22 \cdot I \\
24 \cdot I(22)\end{array}$ \\
\hline & PBS-I & $\begin{array}{l}i l v^{+} \\
\text {phe }\end{array}$ & $\begin{array}{l}1217 \\
1660\end{array}$ & $\begin{array}{l}\text { phe ilv } v^{+} \\
\text {phe } e^{+} i l v\end{array}$ & $\begin{array}{l}288 \\
390\end{array}$ & $\begin{array}{l}23 \cdot 6 \\
23 \cdot 5\end{array}$ \\
\hline \multirow[t]{2}{*}{$\mathrm{SZ}_{4}(\operatorname{argA2} \mathrm{leu}-\mathrm{I})$} & $3 \mathrm{NT}$ & $\underset{\operatorname{leu}^{+} \dagger}{\mathrm{arg}^{+}}$ & $\begin{array}{r}1138 \\
570\end{array}$ & $\begin{array}{l}\text { leuarg } \\
\text { leu+ }\end{array}$ & $\begin{array}{l}809 \\
373\end{array}$ & $\begin{array}{l}71 \cdot 1(73) \\
65 \cdot 4(75)\end{array}$ \\
\hline & \multicolumn{6}{|c|}{$\frac{\text { no. of recombinant colonies }}{\text { total no. of transductants }} \times 100$. } \\
\hline
\end{tabular}

To establish the linkage between different standard markers and hemAI in the recipients, a series of crosses were made with phage $3 \mathrm{NT}$ propagated either on prototrophic or on $\mathrm{SZ} 3$ donor bacteria. The results of these two-point crosses appear in Table 3. Agreement between the percentage recombination values obtained with either of the two selected markers was satisfactory in all cases except when strain sz 6 was the recipient. The same number of bacteria from the same transduction mixture was plated on each selective medium, but there was a difference between the total number of transductants obtained in each case. In contrast, the number of $\mathrm{arg}^{+} \mathrm{hem}^{+}$transductants was almost identical on both media. Either the hem $\mathrm{AI}^{+}$marker alone of the donor did not recombine with the recipient chromosome, or if it did, some of the recombinants were unable to form colonies. The phenomenon was not studied further, and an $88.4 \%$ recombination value was taken for the sz $6 \times \mathrm{sz} 2$ cross in constructing a linkage map. 


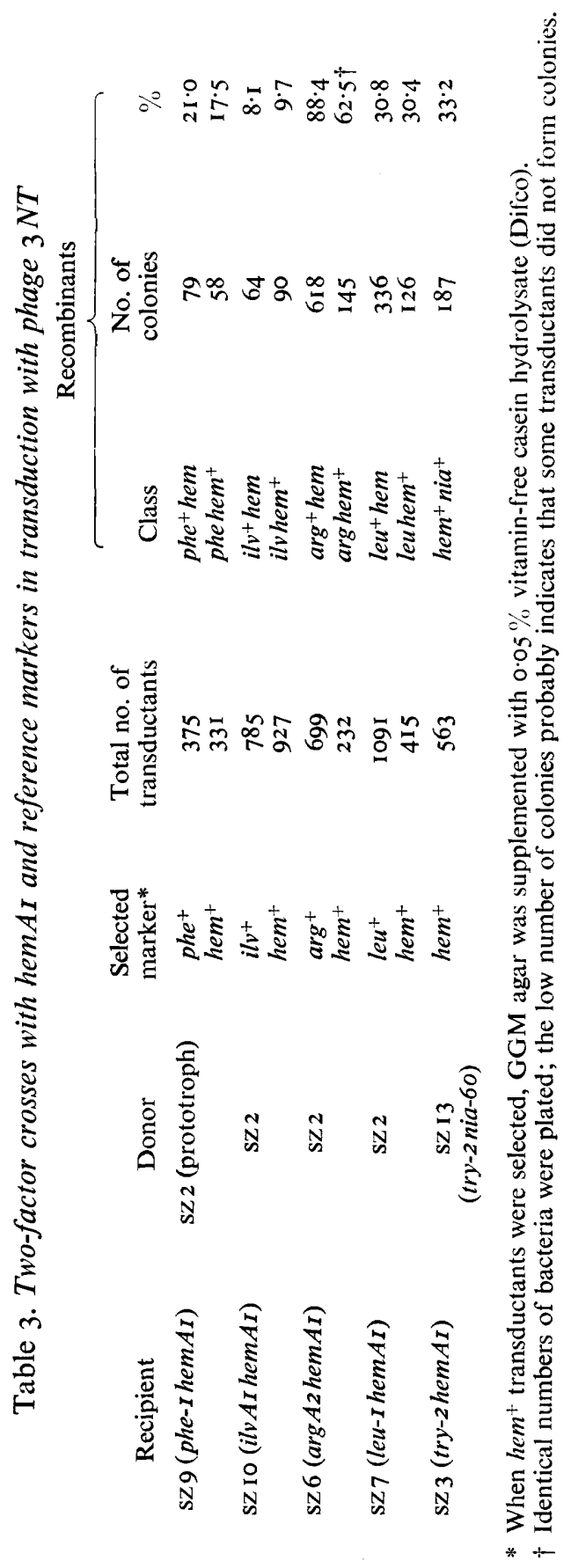


The results of two-factor crosses gave some information on the distance between individual markers and also gave an indication of their sequence, but the markers studied could not be ordered solely on the basis of this data. Consequently a series of three-factor crosses were performed.

Three-factor crosses. In all, six markers were examined in three separate experiments, the results of which are presented in Table 4. In three-factor crosses the class with the lowest number of colonies was taken to represent recombinants in which four cross-overs had occurred. A diagrammatic representation of the possible order of markers in the case of four cross-overs is shown in Fig. I.
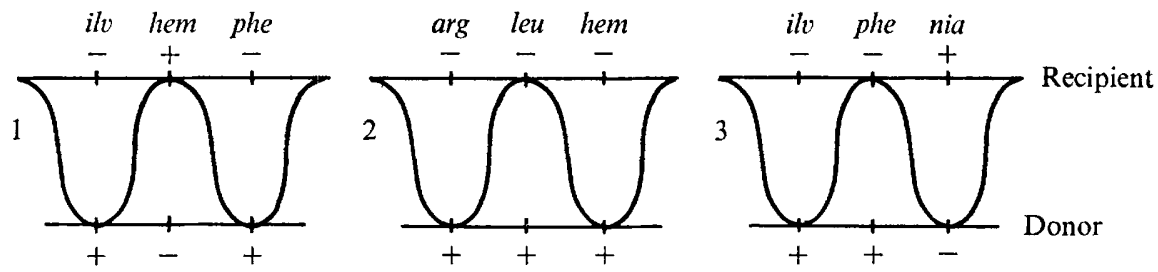

Fig. I. The classes of recombinants involving four cross-overs according to the sequence of markers in the three-point tests of Table 4.

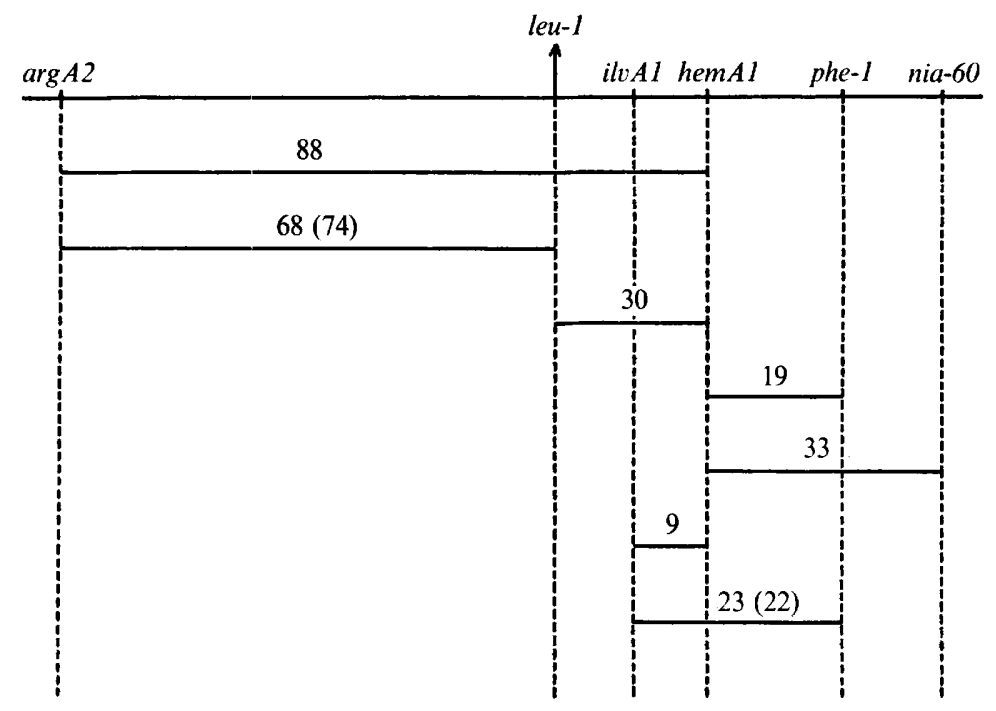

Fig. 2. The position of hemAI locus in linkage group III of Bacillus subtilis.

The results of Expt. I (Table 4) show that hemAI is located between ilvAI and phe-I, while Expt. 3 indicates that phe-I is located between ilvAI and nia-6o. This is in agreement with the results of the two-point crosses (Table 3) and suggests the order ilvAI-hemAIphe-I-nia-6o. On the basis of Expt. 2 and the results of the two-factor crosses, i.e. $\mathrm{sZ}_{4} \times \mathrm{SZ}_{2}$, $\mathrm{SZ} 7 \times \mathrm{SZ} 2$, and SZ6 $\times \mathrm{SZ} 2$, carried out with phage $3 \mathrm{NT}$ (Tables 3 , 4) leu-I seems to be located between argaAz and hemAI. The first marker in linkage group III of Bacillus subtilis I68 is arg according to Dubnau et al. (1967) and $\arg A 2$ is weakly linked to hemAI (see sz $6 \times \mathrm{sz} 2$, Table 3). The results of crosses $\mathrm{SZ} 7 \times \mathrm{SZ} 2$ and sZ $10 \times \mathrm{SZ} 2$ indicate that $i l v A I$ is nearer to hemAI than leu-I is to hemAI. The results of two and three-factor crosses combined suggest 

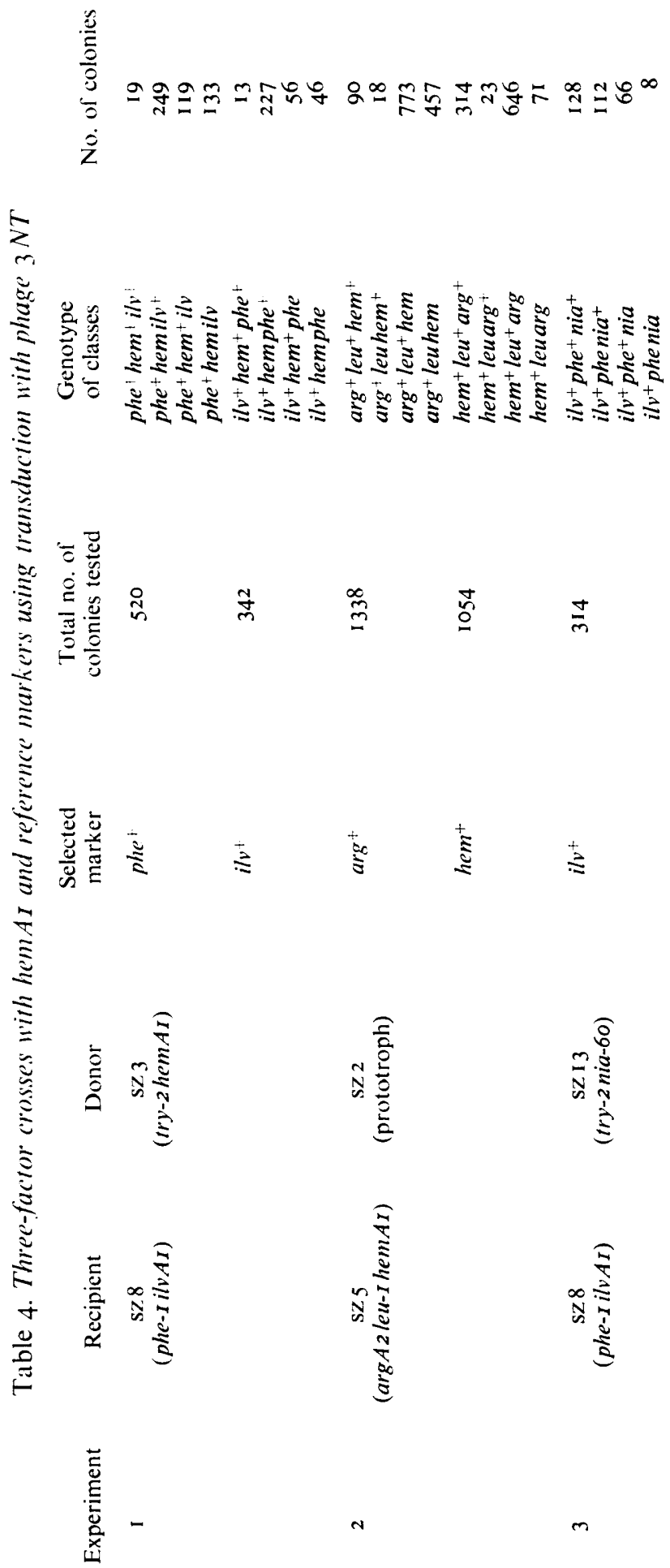


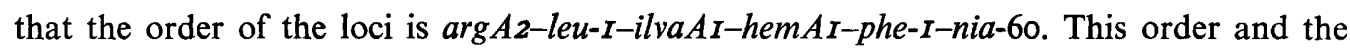
recombination values for the reference markers are in good agreement with those described by Dubnau et al. (1967) in constructing a genetic map of $B$. subtilis 168 . Thus the position of the hemAI gene controlling $\delta$-aminolaevulinic acid synthetase was located in linkage group III between ilvAI and phe-I (Fig. 2). The nia-6o locus of strain SZ I3 is located to the right of the other markers studied. Whether the nia-6o marker or our mutant was identical to the nia markers of Dubnau et al. (1967) is uncertain, but its position is similar.

The authors wish to thank Dr C. Anagnostopoulos, Laboratoire de Génétique et Physiologique, Gif-sur-Yvette, France, and Dr D. Dubnau, Public Health Research Institute, New York, U.S.A., for providing bacterial strains. We are indebted also to Dr J. McGeachie, Royal Infirmary, Glasgow, for help in preparing the manuscript.

\section{REFERENCES}

ANDERson, T. J. \& IvÁNovics, G. (1967). Isolation and some characteristics of haemin dependent mutants of Bacillus subtilis. Journal of General Microbiology 49, 3I-40.

Csiszár, K. \& IvÁnovics, G. (1965). Transduction in Bacillus subtilis. Acta microbiologica Academiae scientiarum hungaricae 12, 73-89.

Dubnau, D., Goldthwaite, C., Smith, I. \& Marmur, J. (1967). Genetic mapping in Bacillus subtilis. Journal of Molecular Biology 27, 163-185.

GoRINI, L. \& KATAJA, E. (1964). Phenotypic repair by streptomycin of defective genotypes in Escherichia coli. Proceedings of the National Academy of Sciences of the United States of America 5I, 486-493.

Hoch, J. A., Barat, M. \& ANAGnostopoulos, C. (1967). Transformation and transduction in recombination-defective mutants of Bacillus subtilis. Journal of Bacteriology 93, 1925-1937.

IvÁNovics, G. \& CsiszÁr, K. (1962). Isolation and some characteristics of Bacillus subtilis phages with transduction activity. Acta microbiologica Academiae scientiarum hungaricae 9, 209-218.

MarJaI, E., KISS, I. \& IVÁNovics, G. (I970). Auxotrophic mutation with low streptomycin resistance and slow growth in Bacillus subtilis. Acta microbiologica Academiae scientiarum hungaricae 17, 133-145.

TAKAHASHI, I. (1961). Genetic transduction in Bacillus subtilis. Biochemical and Biophysical Research Communications $5,171-175$. 